\title{
Molecular mechanisms of Trypanosoma cruzi infection by oral route
}

\author{
Nobuko Yoshida \\ Departamento de Microbiologia, Imunologia e Parasitologia, Universidade Federal de São Paulo, R. Botucatu 862/6², 4023-062 \\ São Paulo, SP, Brasil
}

Frequent reports on outbreaks of acute Chagas' disease by ingestion of food contaminated with parasites from triatomine insects illustrate the importance of this mode of transmission. Studies on oral Trypanosoma cruzi infection in mice have indicated that metacyclic trypomastigotes invade the gastric mucosal epithelium. A key molecule in this process is gp82, a stage-specific surface glycoprotein that binds to both gastric mucin and to target epithelial cells. By triggering $\mathrm{Ca}^{2+}$ signalling, gp82 promotes parasite internalisation. Gp82 is relatively resistant to peptic digestion at acidic $\mathrm{pH}$, thus preserving the properties critical for oral infection. The infection process is also influenced by gp90, a metacyclic stage-specific molecule that negatively regulates the invasion process. T. cruzi strains expressing high gp90 levels invade cells poorly in vitro. However, their infectivity by oral route varies considerably due to varying susceptibilities of different gp 90 isoforms to peptic digestion. Parasites expressing pepsin-susceptible gp90 become highly invasive against target cells upon contact with gastric juice. Such is the case of a T. cruzi isolate from an acute case of orally acquired Chagas' disease; the gp90 from this strain is extensively degraded upon short period of parasite permanence in the gastric milieu. If such an exacerbation of infectivity occurs in humans, it may be responsible for the severity of Chagas' disease reported in outbreaks of oral infection.

Key words: Trypanosoma cruzi - oral infection - metacyclic trypomastigotes - surface molecules - gp 82 - gp90

In the past 20 years transmission of Trypanosoma cruzi, the causative agent of Chagas' disease, has steeply declined in Southern Cone primarily due to control measures directed at the elimination of the domiciliary vector, Triatoma infestans. Notwithstanding the successful elimination of $T$. cruzi transmission by $T$. infestans in Brazil, Uruguay, Chile and substantial areas of Argentina, Bolivia and Paraguay (Schofield et al. 2006), many challenges remain. Increases in transmission have been reported in some areas of Argentina (Gürtler et al. 2005), Venezuela (Feliciangeli et al. 2003) and in the Brazilian Amazon (Coura 2006). Several countries, including Mexico, Peru, Colombia and Costa Rica, have not implemented a national programme for the control of T. cruzi transmission (Schofield et al. 2006). A matter of concern is also the report that wild populations of $T$. infestans are much more widespread throughout Bolivia than predicted, raising the possibility of a threat of reinfestations of treated areas and the eventual spread to neighbouring regions (Noireau et al. 2005).

T. cruzi acquisition via the oral route has recently been the focus of attention. In Brazil, after the control of $T$. cruzi transmission through $T$. infestans and by blood transfusion, the most important and frequent mode of transmission is by the oral route (Coura 2006). Out-

Financial support: FAPESP, CNPq

Corresponding author: nyoshida@unifesp.br

Received 16 March 2009

Accepted 8 May 2009 breaks of acute Chagas' disease through food contamination have occurred in distinct regions of the country in the last four years. The occurrences have been more prevalent in the Amazon Region, where triatomines other than T. infestans predominate. According to Coura et al. (2002), more than $50 \%$ of acute cases of Chagas' disease registered in the Brazilian Amazon between 1968-2000 were attributable to microepidemics of orally transmitted infection.

Studies in mice have shown that insect-derived metacyclic trypomastigotes invade the gastric mucosal epithelium where the parasite replicates as amastigotes; no evidence of parasitic invasion anywhere within the oropharynx or oesophagus has been found (Hoft et al. 1996). Metacyclic forms express mucin-like surface glycoproteins that are highly resistant to proteolytic degradation (Mortara et al. 1992) and enable them to resist the harsh conditions in the stomach. This resistance to proteolysis of the mucins allows the metacyclic forms to proceed towards the target cells located in the gastric mucosa (Yoshida 2006), which constitute the unique portal of entry to systemic infection (Hoft 1996). Contact with gastric juice does not diminish the parasite's infectivity. In fact, some T. cruzi strains may become more invasive upon transient residence in the gastric milieu (Cortez et al. 2006b, Covarrubias et al. 2007). This report summarises recent findings on the molecular mechanisms underlying the process of gastric mucosal infection by metacyclic trypomastigotes in a mouse model. Focused are also the questions of the modulation of T. cruzi infectivity by stomach components, which may either function as barriers to progression towards target cells or may act on parasites to render them more competent for invasion. This potential of stomach components to promote inva- 
sion may thus provide an explanation for the severity of Chagas' disease in orally acquired infection.

\section{Metacyclic stage-specific surface molecule gp82 promotes $T$. cruzi infection by oral route}

Experiments on oral infection in mice using metacyclic trypomastigotes generated in culture have indicated that the stage-specific surface glycoprotein gp82 plays a key role in the invasion of the gastric mucosal epithelium (Neira et al. 2003). Gp82 of different $T$. cruzi strains resists degradation by pepsin at acidic $\mathrm{pH}$, so that the parasites recovered from the stomach $1 \mathrm{~h}$ after oral inoculation into mice, preserve full infectivity (Cortez et al. 2006b). The process of gp82-dependent host cell invasion by metacyclic forms has been partially elucidated by in vitro studies with human epithelial HeLa cells. Parasite-host cell interaction mediated by gp 82 triggers in both cells signal transduction pathways that lead to intracellular $\mathrm{Ca}^{2+}$ mobilisation (Ruiz et al. 1998), an event essential for T. cruzi internalisation (Docampo \& Moreno 1996, Burleigh \& Andrews 1998, Yoshida 2006). In the parasites, the $\mathrm{Ca}^{2+}$ response is induced upon recognition of gp 82 by an as yet undefined host cell receptor expressed in mammalian cells that are susceptible to $T$. cruzi infection, such as HeLa and Vero cells, but not in T. cruzi-resistant cells such as the human leukaemia K562 cells (Yoshida et al. 2000). The events upstream of $\mathrm{Ca}^{2+}$ mobilization appear to involve the activation of the protein tyrosine kinase and phosphorylation of $\mathrm{p} 175$, a protein that is undetectable in noninfective epimastigotes (Favoreto et al. 1998) and activation of phospholipase C. These pathways lead to the generation of inositol 1,4,5-triphosphate ( $\mathrm{IP}_{3}$ ), which promotes $\mathrm{Ca}^{2+}$ release from $\mathrm{IP}_{3}$-sensitive stores (Yoshida et al. 2000). In host cells, phosphoinositide-3 kinase and protein kinase $\mathrm{C}$ appear to be part of the signalling cascades that culminate in $\mathrm{Ca}^{2+}$ mobilization from thapsigargin-susceptible compartments (Ferreira et al. 2006). $\mathrm{Ca}^{2+}$-dependent disorganization of the target cell actin cytoskeleton results from gp82-mediated parasite entry (Cortez et al. 2006a). This may contribute to efficient infection, provided that microfilament rearrangement facilitates T. cruzi internalisation (Rodriguez et al. 1995). Several pieces of evidence indicate that bidirectional signalling triggered by gp 82 ensures the most effective target cell invasion. Metacyclic forms of clone CL-14, for instance, which express gp82 at low levels but otherwise exhibit a surface profile indistinguishable from the highly invasive parental CL strain, invade cultured HeLa cells poorly (Atayde et al. 2004).

Gp82 is encoded by a multigene family that is part of the trans-sialidase/gp 85 superfamily, which is expressed at the infective trypomastigote stage (Araya et al. 1994). It is a glycoprotein that contains N-linked oligosaccharides (Ramirez et al. 1993); however, the carbohydrate portion of the molecule is not involved in host cell binding. Therefore, the recombinant protein based on gp 82 generated in bacteria has the same cell binding capacity as its endogenous counterpart (Ruiz et al. 1998). The gp82 molecule that has been identified by the monoclonal antibody (MAb) 3F6, which inhibits infection in vitro and in vivo when parasites are orally administered, is highly conserved between genetically divergent $T$. cruzi strains. Analysis of the amino acid sequences of gp 82 , as deduced from cDNA clones of G and CL strain metacyclic forms, has revealed an overall identity of $97.9 \%$ and of $100 \%$ within the central domain containing the target cell binding site (Ruiz 1998). At the carboxy-terminal end of the central domain, the cell binding site for gp 82 is contiguous to, and partially overlapping, the epitope for MAb 3F6 (Manque et al. 2000). In addition to the cell invasion promoting activity, gp 82 also has the ability to bind to gastric mucin.

Gastric epithelia are protected by an extracellular mucous layer whose major components are the high molecular mass mucins. During oral T. cruzi infection, the metacyclic trypomastigotes, upon reaching the stomach, bind to gastric mucin through gp82 as a first step towards their destination, i.e., the mucosal epithelial cells. There have been reports that suggest that gp 82 binds selectively to gastric mucin and not, for instance, to mucin from submaxillary glands. This specificity may explain why, in orally inoculated mice, parasites are not found in the oropharynx or oesophagus (Hoft et al. 1996). Such selectivity may determine the stomach cells as the portal of $T$. cruzi entry similar to the preferential adherence to colonic mucin of Shigella dysenteriae, whose pathogenic potential is correlated with its ability to invade and multiply within the cells of colonic epithelium (Sudha et al. 2001). The importance of the gastric mucin-binding property of gp 82 in the establishment of T. cruzi infection by the oral route has been confirmed by studies that have used parasite strains that were deficient in the expression of gp82. Metacyclic forms of gp82-deficient strains express the surface glycoprotein gp30 which induces $\mathrm{Ca}^{2+}$ signalling in host cells and is also recognized by MAb 3F6 (Cortez et al. 2003). The lack of gp82 expression does not affect the parasite's capacity to enter host cells in vitro, presumably because gp30 can fulfil the invasion-promoting function of gp82. Since the invasion of the target cell by gp82-deficient parasites is inhibited to the same extent by either purified native gp30 or by the recombinant gp 82 , it suggests that the same receptor may be recognizing these molecular species (Cortez et al. 2003).

While the in vitro infectivity of gp82-deficient $T$. cruzi strains is similar to that of the gp82-expressing CL strain, the course of infection upon oral administration in mice differs markedly. Gp82-deficient parasites produce very low parasitaemia levels, which may be attributed to the poor affinity of gp30 for gastric mucin (Cortez et al. 2003). Studies that have used systems that mimic the in vivo conditions have demonstrated that poor adhesion to gastric mucin can interfere with parasite entry into subjacent epithelial cells. In the presence of gastric mucin, penetration of gp82-deficient metacyclic trypomastigotes into HeLa cells was significantly reduced while the infectivity of gp 82 expressing strains remained unaltered (Cortez et al. 2003).

Based on the findings from experiments in mice and in cultured epithelial cells, using CL and gp82-deficient strains, the following picture is envisaged (Fig. 1). Upon oral inoculation, metacyclic forms reach the stomach lu- 
A

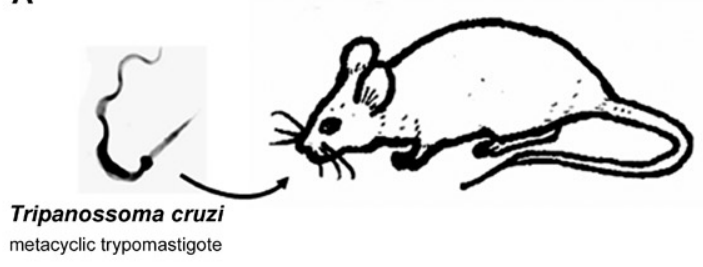

B

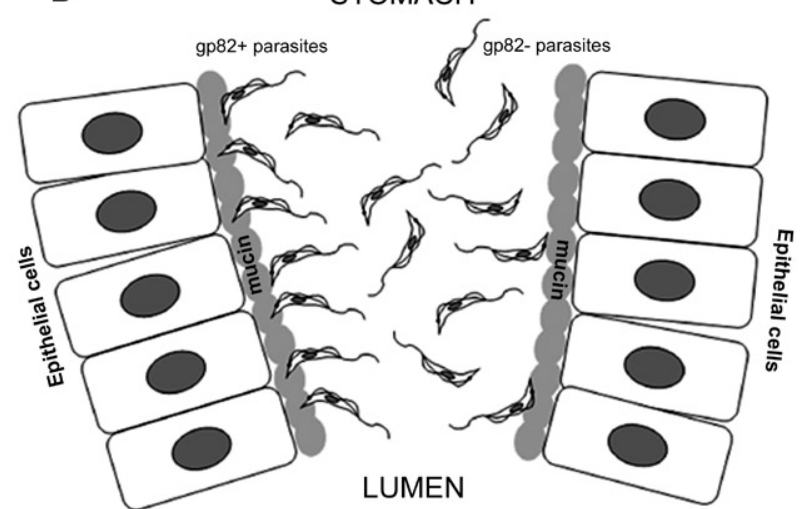

Fig. 1: interaction of $T$. cruzi with gastric mucin in oral infection. Upon inoculation into mice (A), gp82-expressing and gp82-deficient metacyclic trypomastigotes reach the stomach lumen (B). Parasites expressing gp 82 bind to gastric mucin in the mucous layer by engaging this surface molecule, as a first step towards underlying epithelial cells. By contrast, a few gp82-deficient parasites bind to gastric mucin, possibly using gp30.

men, where the gp82-expressing parasites bind to gastric mucin as an initial step for translocation towards the target epithelial cells; this process appears to require parasite energy. For metacyclic forms of gp82-deficient T. cruzi strains, which adhere, albeit poorly, to gastric mucin in gp30-mediated fashion, the mucus layer constitutes a barrier that prevents most parasites from reaching the mucosal epithelium. Following the initial binding, the process of cell invasion is initiated (Fig. 2). Metacyclic forms that express gp 82 engage this molecule to accomplish the internalisation process by triggering the signalling cascades that lead to an increase in intracellular $\mathrm{Ca}^{2+}$ concentration. Gp82-deficient metacyclic forms that manage to traverse the mucus layer enter host cells in a gp30-mediated manner, thus inducing $\mathrm{Ca}^{2+}$ mobilization. After $\sim 12 \mathrm{~h}$ post-invasion, most metacyclic forms have transformed into amastigotes, which go through replication cycles. By $96 \mathrm{~h}$, nests of amastigotes are detectable at much higher numbers in the histological preparations of the stomach of mice infected with gp82-expressing parasites than in sections from animals that were inoculated with gp82-deficient strains (Fig. 3). There is no difference between strains with regards to the rate of intracellular multiplication so that the release of trypomastigotes into the circulation should occur within a comparable time frame. However, reflecting the difference in the rate of invasion, parasitaemia levels in mice infected with gp82-deficient parasites are much lower than in mice inoculated with gp82-expressing parasites.

It should be noted that the CL strain and the strains deficient in gp82 expression referred in this report express gp90 molecule at low levels (Cortez et al 2003). Therefore their infectivity is not influenced by this molecule. Gp90 is a metacyclic, stage-specific surface glycoprotein (Teixeira \& Yoshida 1986) that is ubiquitously present in the T. cruzi population (Yoshida 2006). This surface molecule binds to mammalian cells in a receptormediated manner without inducing $\mathrm{Ca}^{2+}$ response (Ruiz et al. 1998) and therefore acts as a negative regulator of target cell invasion (Málaga \& Yoshida 2001).

\section{Susceptibility of gp90 molecule to proteolysis influ- ences $T$. cruzi infectivity by oral route}

Expression of gp90, the metacyclic stage-specific surface glycoprotein that down modulates parasite infectivity (Málaga \& Yoshida 2001), varies in different T. cruzi strains and its expression in a strain is inversely correlated with the ability of that strain to invade cul-

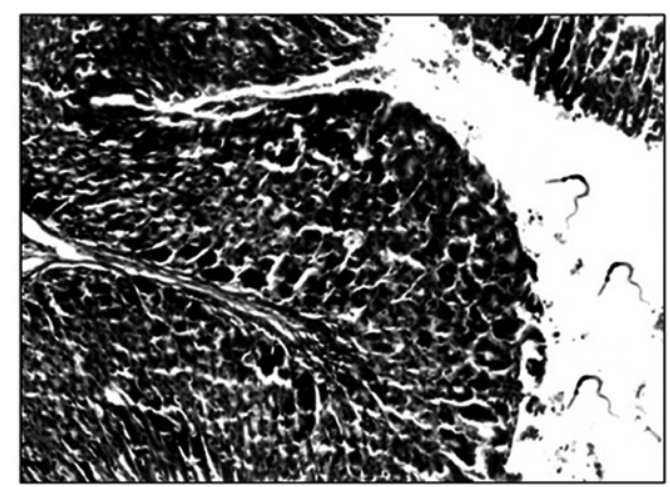

STOMACH

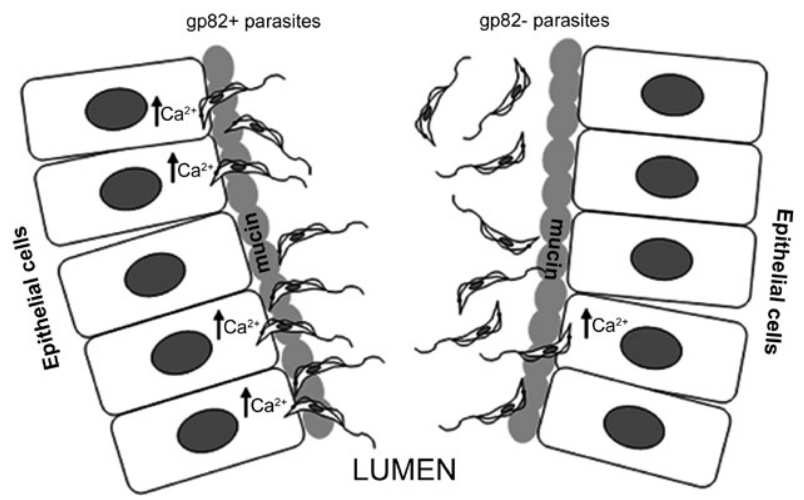

Fig. 2: penetration of Trypanosoma cruzi into gastric epithelial cells in oral infection. Upon reaching the target cells, gp82-expressing metacyclic forms accomplish the invasion process by triggering the gp82-mediated signaling cascades that lead to an increase in intracellular $\mathrm{Ca}^{2+}$ concentration. Metacyclic forms of gp82-deficient T. cruzi strains that managed to traverse the mucus layer enter host cells in gp30-mediated manner, inducing $\mathrm{Ca}^{2+}$ mobilization. The upper part of the figure is a composition of a histological section of mouse stomach stained by hematoxylin-eosin and Giemsa-stained metacyclic trypomastigotes that are not in the same scale. 


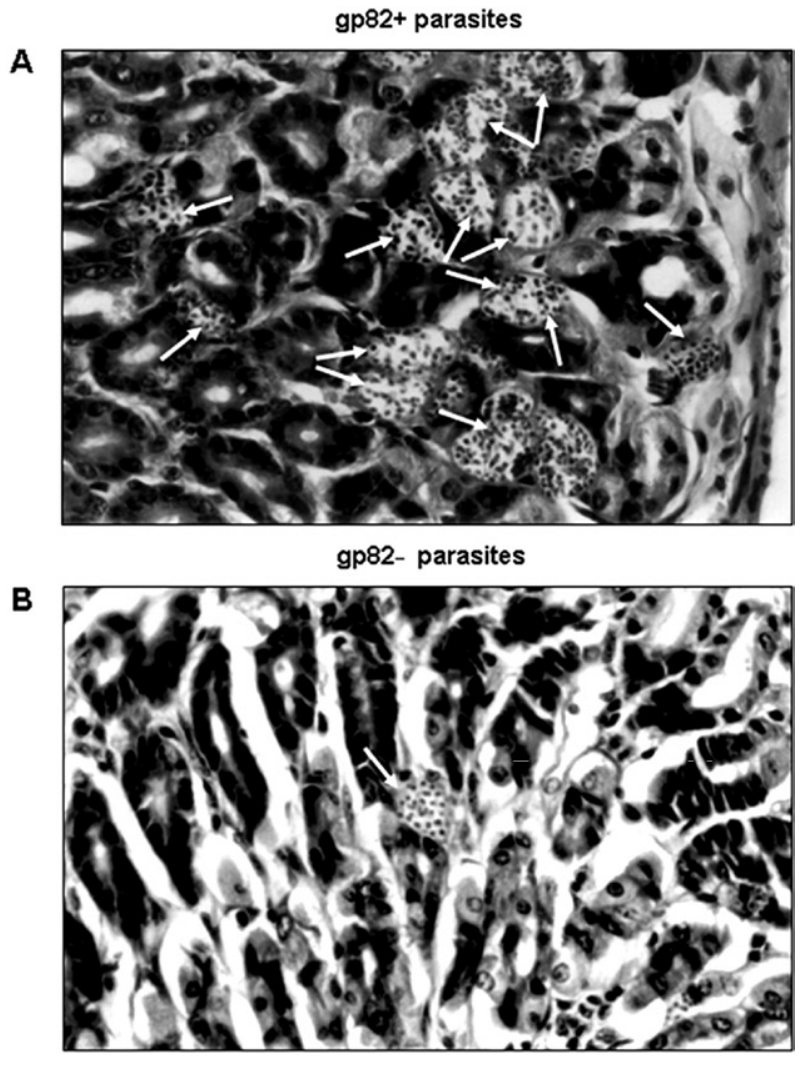

Fig. 3: intracellular replication of Trypanosoma cruzi in gastric mucosal epithelium. Following oral inoculation of metacyclic trypomastigotes into mice, the parasites invade target cells in the stomach and replicate as amastigotes. Shown are the histological sections of the mouse stomach stained by hematoxylin-eosin four days post-infection. Nests of replicating amastigotes (white arrows) can be seen in higher numbers in mice infected with gp82-expressing parasites (A) than in animal inoculated with gp82-deficient strains (B).

tured mammalian cells (Ruiz et al. 1998, Yoshida 2006). Two variant forms of gp90 have been identified based on the reactivity with MAbs 1G7 and/or 5E7 (Teixeira $\&$ Yoshida 1986). The complete peptide sequence of either of these gp90 molecules remains to be determined. Attempts to clone the gene encoding gp90 have, so far, been unsuccessful. What is known is that the sequence is present in multiple copies in the genome encodes a gp90 carboxy-terminal portion containing the epitope for MAb 5E7 (Franco et al. 1993), an antibody that reacts with metacyclic forms of all T. cruzi strains examined to date (Yoshida 2006). The recombinant protein corresponding to the carboxy-terminal domain of gp90 binds to target cells in a receptor-mediated manner that is indistinguishable from that of native gp90 (Ruiz et al. 1998). However, the receptors for the recombinant and the native molecules may be distinct. MAb 1G7, which does not react with the recombinant protein, binds to live metacyclic trypomastigotes and inhibits parasite-induced intracellular $\mathrm{Ca}^{2+}$ mobilization (Ruiz et al. 1998). Thus, the gp90-reactive MAb 1G7 has an opposing effect to that of gp82-reactive MAb 3F6, which triggers an increase in cytosolic $\mathrm{Ca}^{2+}$ concentration upon binding to metacyclic forms (Ruiz et al. 1998). Of interest is that while MAb 3F6 induces the activation of the protein tyrosine kinase and phosphorylation of 175 , (Favoreto et al. 1998), MAb 1G7 promotes p175 dephosphorylation (Manque et al. 2003). These findings are compatible with the idea that activation of protein tyrosine kinases and intracellular $\mathrm{Ca}^{2+}$ mobilization are associated events (Yoshida et al. 2000).

Metacyclic forms of T. cruzi strains expressing gp 90 at high levels invade cultured cells poorly. However, it is not possible to predict from this in vitro behaviour the course of infection after oral administration into mice. Recent studies have indicated that in vivo infectivity of these parasites is determined by gp 90 susceptibility to peptic digestion. This notion is illustrated in the case of two T. cruzi strains that were derived from patients during the acute phase of the disease (Fig. 4). Metacyclic forms of these strains, which exhibited a similar surface profile (with high expression of gp82, gp30 and gp90), entered cultured epithelial cells at low numbers (Cortez et al. 2006b). This provides evidence that gp90mediated parasite-host cell interactions predominate over those interactions that are mediated by the gp 82 or gp30 molecules. When orally inoculated into mice, the two strains exhibited distinct infective properties. One of these strains entered gastric mucosal epithelial

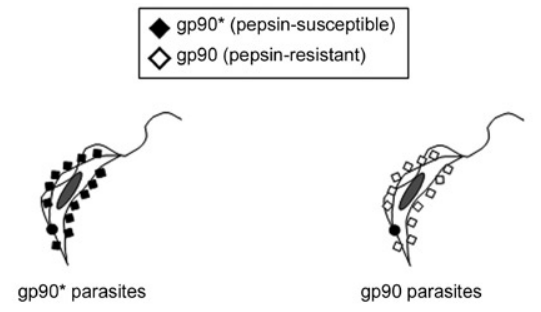

STOMACH

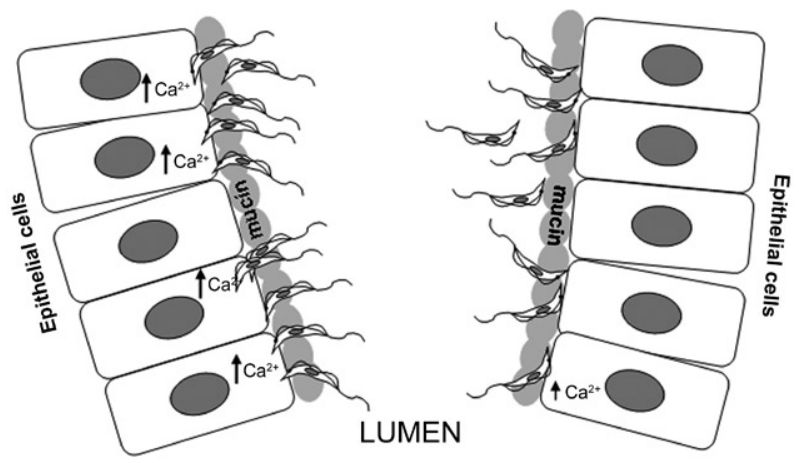

Fig. 4: increased infectivity of Trypanosoma cruzi strains expressing gp90 molecules susceptible to peptic digestion. Upon oral infection, metacyclic trypomastigotes of parasite strains bearing pepsinsusceptible gp $90 *$ molecules express much less of these cell invasion down modulators, so that they can enter target cells more effectively. By contrast, parasites bearing pepsin-resistant gp 90 molecules bind to target cells and relay inhibitory signal to both cells, thus hampering parasite entry. 
cells in high numbers and, as a consequence, resulted in a high number of replicating parasites in amastigote nests by day 4 as determined by haematoxylin-eosin stained histological sections of the stomach. At later times, the infected mice developed high parasitaemias and about $30 \%$ of them died within 30 days of infection. By analysing the parasites recovered from the mouse stomach $1 \mathrm{~h}$ after oral infection, the gp90 molecules was found to be extensively degraded, in a manner similar to in vitro treatment of parasites with pepsin at acidic $\mathrm{pH}$. Metacyclic forms with digested gp90, either upon transient residence in the gastric milieu or upon pepsin treatment in vitro, displayed an increased capacity to enter target cells (Cortez et al. 2006b). In contrast with these observations, mice inoculated with the second $T$. cruzi strain developed very low parasitaemias. Consistent with this, at day 4 post-inoculation the number of amastigote nests found in the stomach was very low and mortality was null. Analysis of parasites recovered from the mouse stomach $1 \mathrm{~h}$ after oral infection showed that the gp90 molecule remained intact, thus explaining why metacyclic forms of this strain are as poorly infective in oral infection as they are towards cultured epithelial cells. It should be noted that, apart from the differential susceptibility of gp90 to peptic digestion, the two T. cruzi strains did not exhibit marked differences, with both strains preserving fully functional gp 82 and gp 30 molecules after being recovered from the gastric milieu (Cortez et al. 2006b).

Overall, these findings suggest that the severity of acute Chagas' disease that has been reported in patients infected by the oral route may be associated with upregulation of parasite infectivity, due to digestion of the cell invasion inhibitor, gp90. Support for this hypothesis has been provided by the first study of a T. cruzi isolate derived from an acute case of Chagas' disease acquired by oral route. This isolate, from an outbreak of oral T. cruzi infection that occurred in the southern state of Santa Catarina in 2005 and resulted in three deaths, was designated SC. Metacyclic trypomastigotes of SC, characterized by high levels of expression of gp 82, gp30 and gp90, entered host cells in culture at low rates (Covarrubias et al. 2007). When inoculated orally into mice, however, the parasites produced high parasitaemias and high mortality with more than $50 \%$ of infected animals being dead by day 20 post-inoculation. Analysis of SC parasites recovered from the mouse stomach $1 \mathrm{~h}$ after oral administration failed to detect the presence of gp90 molecule, indicating that it was completely digested upon contact with the gastric juice. However, while the amino-terminal portion of gp82 was digested, the domain implicated in gastric mucin-binding and host cell invasion was fully preserved. With a fully functional gp82, in addition to a pepsin-resistant gp30, the internalisation of the parasite increased.

\section{Do $T$. cruzi gp35/50 molecules play a role in oral infection?}

T. cruzi strains referred to in preceding sections, derived mostly from chagasic patients, rely to a certain extent on gp82 or gp30 to establish infection in mice by oral route. For these strains, mucin-like surface glycoproteins primarily serve to protect the parasites against the action of gastric juice. However, gp35/50 mucins have an additional role in $T$. cruzi strains that preferentially engage these molecules to invade target cells in vitro. Among T. cruzi strains examined to date, two groups have emerged based on the expression of mucinlike gp35/50 glycoproteins: those reacting solely with $\mathrm{MAb} 2 \mathrm{~B} 10$ and those reacting with both MAb 2B10 and MAb 10D8. Strains from human origin that have been studied so far express gp35/50 molecules reactive with MAb 2B10 but not with MAb 10D8, whereas most strains derived from wild animals or wild triatomine insects express MAb 10D8-reactive gp35/50 (Mortara et al. 1992). The gp35/50 mucins are encoded by a large multigene family (Di Noia et al. 1998) and are expressed in metacyclic forms as well as in epimastigotes (Yoshida et al. 1989). These mucins are GPI-anchored glycoproteins rich in threonine residues. In the protein core, glycans are $\mathrm{O}$-linked to the threonine residues through $\mathrm{N}$ acetylglucosamine rather than $\mathrm{N}$-acetylgalactosamines as usually found in vertebrate mucins. (Schenkman et al. 1993). Depending on the T. cruzi strain, they may contain galactofuranose residues in addition to galactopyranose (Previato et al. 1994, Acosta-Serrano et al. 1995, Salto et al. 2000). MAb 10D8 reacts with gp35/50 epitopes containing galactofuranose, whereas MAb 2B10 appears to recognize galactopyranose-containing epitopes present in all strains. Several lines of evidence have indicated that MAb 10D8-reactive gp35/50 is implicated in host cell invasion as both the antibody and the purified mucin are capable of inhibiting parasite internalisation (Yoshida et al. 1989, Ruiz et al. 1993). Gp35/50 glycoprotein binds to target cells in a receptor-mediated manner and induces a bidirectional $\mathrm{Ca}^{2+}$ response, but to a lesser degree than gp82 (Ruiz et al. 1998). It is possible that most parasite strains that rely preferentially on gp $35 / 50$ to enter host cells are poorly invasive because of the relatively weak signalling activity of these molecules. However, the low invasive capacity may also be due to the expression of gp90, which in general is present at high levels in metacyclic forms of $T$. cruzi strains that are recognized by MAb 10D8 (Yoshida 2006). As these strains also exhibit high expression of gp82, the results support the hypothesis that the gp90-mediated interaction with the host cell is dominant acting. Gp35/50 mucins contain sialic acid but sialyl residues are not required for invasion and, depending on the parasite strain, they may even impair recognition by target cells (Yoshida et al. 1997). For instance, treatment of metacyclic forms of $\mathrm{G}$ strain with bacterial neuraminidase removes gp35/50 sialic acid and increases the parasite infectivity (Yoshida et al. 1997). This can be reverted by resialylation of gp35/50 upon incubating the parasites with $T$. cruzi trans-sialidase and sialyl lactose, which results in parasite internalisation to similar levels as that seen before desialylation. Consistent with this result, the ability of desialylated gp35/50 to bind to target cells and to trigger $\mathrm{Ca}^{2+}$ response was found to be higher than that of its sialylated counterpart (Yoshida et al. 1997).

$\mathrm{G}$ strain metacyclic forms that engage MAb 10D8reactive gp35/550 mucins to enter host cells in vitro are 
poorly infective when given orally into mice and fail to produce detectable parasitaemia upon microscopic examination of blood samples (Yoshida 2006). Analysis of histological preparations of stomach from mice infected with $\mathrm{G}$ strain has revealed very scarce parasites. These observations are consistent with the fact that this strain expresses high levels of a gp90 isoform resistant to peptic digestion (Covarrubias et al. 2007). If metacyclic forms expressing 10D8-reactive gp35/50, but not gp90, could be generated, the role of T. cruzi mucins in infection in vivo may be clarified. Finding proteases and/or conditions that preserve the gp35/50 molecules and degrade gp90 would facilitate researchers to gain insight into the role that mucins play in infection.

Studies on experimental oral infection by T. cruzi metacyclic trypomastigotes, in parallel with in vitro epithelial cell invasion assays, have revealed several features of parasite-host interactions, which would not have been predicted soley based on in vitro experiments. The gastric mucosal epithelium as the preferential portal of entry for metacyclic forms inoculated in mice by the oral route may be due to the selective binding of parasites to the gastric mucin. In the gastric milieu, metacyclic forms resist digestion due to the presence of surface mucin-like gp35/50 glycoproteins. Metacyclic stagespecific surface molecule gp82, implicated in gastric mucin-binding and in promoting host cell invasion, is relatively resistant to peptic digestion and remains fully functional upon transient contact with gastric juice. Gp90, a metacyclic stage-specific surface molecule that functions as a negative regulator of target cell entry and is the main determinant of parasite infectivity, has strain-dependent susceptibility to proteolysis. Metacyclic forms expressing gp82 and gp90 at low levels traverse the mucous layer protecting the gastric mucosa through gp82-mediated binding to its major components, the mucin molecules, and efficiently invade the target epithelial cells engaging gp82. For metacyclic forms deficient in $\mathrm{gp} 82$, the mucous layer represents a barrier that hampers the journey towards host cells. For T. cruzi strains that express the cell invasion-promoting molecules gp82 and gp30, in addition to gp90 at high levels, their infectivity will be determined by the susceptibility of gp90 isoform to peptic digestion. If they express pepsin-resistant gp90, they will be poorly infective. However, if they express pepsin-susceptible gp90 molecules, they can become highly infective upon exposure to gastric juice. The latter situation appears to be the case in a T. cruzi isolate from an acute case of Chagas' disease acquired by oral infection, which had its infectivity enhanced in the mouse gastric milieu.

\section{ACKNOWLEDGEMENTS}

To Dr. Clara Lucia Barbieri and Dr. Rafael Miyazawa Martins, for reading the manuscript.

\section{REFERENCES}

Acosta AS, Schenkman S, Yoshida N, Mehler A, Richardson JM, Ferguson MAJ 1995. The lipid structure of the GPI-anchored mucinlike sialic acid acceptors of Trypanosoma cruzi changes during parasite differentiation from epimastigotes to infective metacyclic trypomastigote forms. J Biol Chem 270: 27244-27253.

Araya JE, Cano MI, Yoshida N, Franco da Silveira J 1994. Cloning and characterization of a gene for the stage-specific $82-\mathrm{kDa}$ surface antigen of metacyclic trypomastigotes of Trypanosoma cruzi. Mol Biochem Parasitol 65: 161-169.

Atayde VD, Neira I, Cortez M, Ferreira D, Freymuller E, Yoshida N 2004. Molecular basis of non virulence of Trypanosoma cruzi clone CL-14. Int J Parasitol 34: 851-860.

Burleigh BA, Andrews NW 1998. Signaling and host cell invasion by Trypanosoma cruzi. Curr Opinion Microbiol 1: 451-465.

Cortez M, Atayde V, Yoshida N 2006a. Host cell invasion mediated by Trypanosoma cruzi surface molecule gp 82 is associated with F-actin disassembly and is inhibited by enteroinvasive Escherichia coli. Microbes Infect 8: 1502-1512.

Cortez M, Neira I, Ferreira D, Luquetti AO, Rassi A, Atayde VD, Yoshida N 2003. Infection by Trypanosoma cruzi metacyclic forms deficient in gp82 but expressing a related surface molecule gp30. Infect Immun 71: 6184-6191.

Cortez M, Silva MR, Neira I, Ferreira D, Sasso GRS, Luquetti AO, Rassi A, Yoshida N 2006b. Trypanosoma cruzi surface molecule gp90 downregulates invasion of gastric mucosal epithelium in orally infected mice. Microbes Infect 8: 36-44.

Coura JR 2006. Transmission of chagasic infection by oral route in the natural history of Chagas' disease. Rev Soc Bras Med Trop 39 (Suppl. 3): 113-117.

Coura JR, Junqueira ACV, Fernandes O, Valente SAS, Miles MA 2002. Emerging Chagas' disease in Amazonian Brazil. Trends Parasitol 18: 171-176.

Covarrubias C, Cortez M, Ferreira D, Yoshida N 2007. Interaction with host factors exacerbate Trypanosoma cruzi cell invasion capacity upon oral infection. Int J Parasitol 37: 1609-1616.

Di Noia JM, D’Orso I, Aslund I, Sanchez DO, Frasch AC 1998. The Trypanosoma cruzi mucin family is transcribed from hundreds of genes having hypervariable regions. J Biol Chem 273: 1084310850 .

Docampo R, Moreno SJ 1996. The role of $\mathrm{Ca}^{2+}$ in the process of cell invasion by intracellular parasites. Parasitol Today 12: 61-65.

Favoreto Jr S, Dorta ML, Yoshida N 1998. Trypanosoma cruzi 175 $\mathrm{kDa}$ protein tyrosine phosphorylation is associated with host cell invasion. Exp Parasitol 89: 188-194.

Feliciangeli MD, Campbell-Lendrum D, Martinez C, Gonzalez D, Coleman P, Davies C 2003. Chagas' disease control in Venezuela: lessons for the Andean Region and beyond. Trends Parasitol 19: 44-49.

Ferreira D, Cortez M, Atayde VD, Yoshida N 2006. Actin cytoskeleton-dependent and independent host cell invasion by Trypanosoma cruzi is mediated by sdistinct parasite surface molecules. Infect Immun 74: 5522-5528.

Franco FRS, Paranhos-Bacalla GS, Yamauchi LM, Yoshida N, Franco da Silveira J 1993. Characterization of a cDNA clone encoding the carboxy-terminal domain of a 90-kilodalton surface antigen of Trypanosoma cruzi metacyclic trypomastigotes. Infect Immun 61: 4196-4201.

Gürtler RE, Cecere MC, Lauricella MA, Petersen RM, Chuit R, Segura EL, Cohen JE 2005. Incidence of Trypanosoma cruzi infection among children following domestic infestation after insecticide spraying in rural Northwestern Argentina. Am J Trop Med Hyg 73: 95-103. 
Hoft DF 1996. Differential mucosal infectivity of different life stages of Trypanosoma cruzi. Am J Trop Med Hyg 55: 360-364.

Hoft DF, Farrar PL, Kratz-Owens K, Shaffer D 1996. Gastric invasion by Trypanosoma cruzi and induction of protective mucosal immune responses. Infect Immun 64: 3800-3810.

Málaga S, Yoshida N 2001. Targeted reduction in expression of Trypanosoma cruzi surface glycoprotein gp90 increases parasite infectivity. Infect Immun 69: 353-359.

Manque PM, Eichinger D, Juliano MA, Juliano L, Araya J, Yoshida N 2000. Characterization of the cell adhesion site of Trypanosoma cruzi metacyclic stage surface glycoprotein gp82. Infect Immun 68: $478-484$.

Manque PM, Neira I, Atayde VD, Cordero E, Ferreira AT, Franco da Silveira J, Ramirez M, Yoshida N 2003. Cell adhesion and $\mathrm{Ca}^{2+}$ signaling activity in stably transfected Trypanosoma cruzi epimastigotes expressing the metacyclic stage-specific surface molecule gp82. Infect Immun 71: 1561-1565.

Mortara RA, Silva S, Araguth MF, Blanco SA, Yoshida N 1992. Polymorphism of the 35- and 50-kilodalton surface glycoconjugates of Trypanosoma cruzi metacyclic trypomastigotes. Infect Immun 60: 4673-4678.

Neira I, Silva FA, Cortez M, Yoshida N 2003. Involvement of Trypanosoma cruzi metacyclic trypomastigote surface molecule gp82 in adhesion to gastric mucin and invasion of epithelial cells. Infect Immun 71: 557-561.

Noireau F, Cortez MGR, Monteiro FA, Jansen AM, Torrico F 2005. Can wild Triatoma infestans foci in Bolívia jeopardize Chagas' disease control efforts? Trends Parasitol 21: 7-10.

Previato JO, Jones C, Gonçalves LP, Wait R, Travassos LR, Mendonça-Previato L 1994. O-glycosidically linke N-acetylglucosaminebound oligosaccharides from glycoproteins of Trypanosoma cruzi. Biochem J 301: 151-159.

Ramirez MI, Ruiz RC, Araya JE, Franco da Silveira J, Yoshida N 1993. Involvement of the stage-specific 82-kilodalton adhesion molecule of Trypanosoma cruzi metacyclic trypomastigotes in host cell invasion. Infect Immun 61: 3636-3641.

Rodriguez A, Rioult MG, Ora A, Andrews NW 1995. A trypanosomesoluble factor induces $\mathrm{IP}_{3}$ formation, intracellular $\mathrm{Ca}^{2+}$ mobilization and microfilament rearrangement in host cells. J Cell Biol 129: $1263-1273$

Ruiz RC 1998. Trypanosoma cruzi: associação entre infectividade e expressão de glicoproteinas com atividade sinalizadora de cálcio, PhD Thesis, Universidade Federal de São Paulo, São Paulo, 107p.

Ruiz RC, Favoreto S, Dorta ML, Oshiro MEM, Ferreira AT, Manque PM, Yoshida N 1998. Infectivity of Trypanosoma cruzi strains is associated with differential expression of surface glycoproteins with differential $\mathrm{Ca}^{2+}$ signaling activity. Biochem J 330: 505-511.

Ruiz RC, Rigoni VLl, Gonzalez J, Yoshida N 1993. The 35/50 kDa surface antigen of Trypanosoma cruzi metacyclic trypomastigotes, an adhesion molecule involved in host cell invasion. Parasite Immunol 15: 121-123.

Salto ML, Gallo-Rodriguez C, Lima C, Lederkremer RM 2000. Separation of galfbeta $1 \rightarrow$ XGlcNAc and galpbeta $\rightarrow$ XGlcNAc $(\mathrm{X}=$ 3,4 and 6 ) as the alditols by high-pH aninon-exchange chromatography and thin-layer chromatography: characterization of mucins from Trypanosoma cruzi. Anal Biochem 279: 79-84.

Schenkman S, Ferguson MAJ, Heise N, Cardoso de Almeida ML, Mortara RA, Yoshida N 1993. Mucin-like glycoproteins linked to the membrane by glycosylphosphatidylinositol anchor are the major acceptors of sialic acid in a reaction catalysed by trans-sialidase in metacyclic forms of Trypanosoma cruzi. Mol Biochem Parasitol 59: 293-304.

Schofield CJ, Jannin J, Salvatella R 2006. The future of Chagas' disease control. Trends Parasitol 22: 583-588.

Sudha PS, Devaraj H, Devaraj N 2001. Adherence of Shigella dysenteriae to human colonic mucin. Curr Microbiol 42: 381-387.

Teixeira MMG, Yoshida N 1986. Stage-specific surface antigens identified by monoclonal antibodies. Mol Biochem Parasitol 18 : 271-282.

Yoshida N 2006. Molecular basis of mammalian cell invasion of Trypanosoma cruzi. An Acad Bras Cienc 78: 87-111.

Yoshida N, Dorta ML, Ferreira AT, Oshiro MEM, Mortara RA, Acosta-Serrano A, Favoreto Jr S 1997. Removal of sialic acid from mucin-like surface molecules of Trypanosoma cruzi metacyclic trypomastigotes enhances parasite-host cell interaction. Mol Biochem Parasitol 84: 57-67.

Yoshida N, Favoreto Jr S, Ferreira AT, Manque PM 2000. Signal transduction induced in Trypanosoma cruzi metacyclic trypomastigotes during the invasion of mammalian cells. Braz J Med Biol Res 33: 269-278.

Yoshida N, Mortara RA, Araguth MF, Gonzalez JC, Russo M 1989. Metacyclic neutralizing effect of monoclonal antibody 10D8 directed to the 35 and 50-kilodalton surface glycoconjugates of Trypanosoma cruzi. Infect Immun 57: 1663-1667. 